\title{
AN EDGE DETECTION MODEL BASED ON NON-LINEAR LAPLACE FILTERING
}

\author{
Lucas J. van VLIET ${ }^{1}$, Ian T. YOUNG ${ }^{1}$, and Guus L. BECKERS ${ }^{2}$ \\ 1) Pattern Recognition Group of the Faculty of Applied Physics \\ Delft University of Technology \\ Lorentzweg 1, 2628 CJ Delft, The Netherlands \\ 2) Department of Medical Informatics \\ Erasmus University \\ Rotterdam, The Netherlands
}

\begin{abstract}
We have developed and evaluated an edge detection scheme using a non-linear Laplace operator and the Marr-Hildreth model of edge detection. The technique is extremely effective and flexible in detecting one-pixel thick edges in images whose signal-to-noise ratios (SNR) range from $40 \mathrm{~dB}$ down to $0 \mathrm{~dB}$. We have compared our results with those in the literature. For the test images we considered, our configuration performs at least as well - and in most cases far better - than other edge detectors. For these comparisons we have used Pratt's figure-of-merit as a quantitative performance measure. At very low signal-to-noise ratios $(<10 \mathrm{~dB})$, our detector is superior to all the others tested and produces closed contours.
\end{abstract}

Specific characterizations of the non-linear Laplacian are its adaptive orientation to the direction of the gradient, its inherent masks which permit the development of approximately circular (isotropic) filters, and its easy and fast implementation in software.

\section{INTRODUCTION}

Edge detection is one of the most important tasks in image processing and scene analysis systems. It is frequently used in scene segmentation, where borders between adjacent regions have to be extracted. During the past two decades edge detection has been a major topic of research providing many algorithms that perform well in a given application, but poorly in most others. The objective of our research is, therefore, to develop an edge detection scheme robust enough to perform well over a wide range of signal-to-noise ratios in various types of images.

We define an edge as:

edge - a simply-connected contour, one pixel thick, at the center of the slope between two adjacent regions with a considerable difference in grey level.

A detector, based on the detection of zero-crossings in the output of a "Laplace" filtered image can produce such edges. At the position of true edges there is a peak in the first derivative perpendicular to the local edge direction. These peaks correspond to zero-crossings in the second derivative along the gradient. The Marr-Hildreth edge detection model, which is based on neurophysiological studies, combines a Laplace filter with a Gaussian smoothing filter (Marr [1]).

L.J. van Vliet, I.T. Young, A.L.D. Beckers, An edge detection model based on nonlinear Laplace filtering, in: Pattern Recognition and Artificial Intelligence, E.S. Gelsema and L.N. Kanal (eds), Elsevier Science Publishers B.V. (North-Holland), 1988, 63-73. 
The size, standard deviation, of the Gaussian filter selects a size scale at which possible changes in grey level are detected. The frequency band to be chosen strongly depends on the noise level and type of input image. The necessity of this combination is confirmed by the revival of the Laplace operator, which had fallen into disuse [2] before Marr's publication.

A non-linear Laplace-like operator in a 3x3 neighborhood proposed by Beckers [3] showed to be a less noise-sensitive alternative to the classic Laplace operator. In our research we have incorporated the non-linear Laplace operator in an extended version of the Marr-Hildreth model for edge detection. The next section describes the different functions of the edge detection scheme. The scheme consists of parallel algorithms, these are algorithms in which the detection of a pixel as an edge element is independent of the results elsewhere in the image. Section 3 describes the evaluation procedure that is used to locally optimize the detector over a wide range of signal-tonoise ratios. Section 4 shows some experimental results achieved with this detector. In section 5, we present a comparative study of this technique with those of others using Pratt's figure of merit as a quantitative performance measure (Pratt [4]). At the end conclusions might be drawn concerning the applicability of the approached technique.

\section{EDGE DETECTION SCHEME}

The Marr-Hildreth model for edge detection consists of a combination of a band pass filter (Gaussian filter) and a high pass filter (Laplace filter) followed by a zero-crossing detector. The resulting Laplacian of a Gaussian $\left(\nabla^{2} \mathrm{G}_{\sigma}(\mathrm{x}, \mathrm{y})\right)$ is often called the 'Mexican-Hat' operator, due to its characteristic shape. The mask can be decomposed into separate row and column filters to increase computational speed on general purpose computers. In our scheme we split the 'Mexican hat' in a smoothing filter and a Laplace filter. Apart from the zero-crossings edge strengths are computed. Finally, these results are combined into one image of which the pixels have a grey value representing the likelihood that they belong to an edge in the original image. In the case the image contains objects and background with a significant difference in grey level, thresholding at a proper level produces closed contours according to our definition of an edge. A flow diagram of the described model is presented by figure 1 .

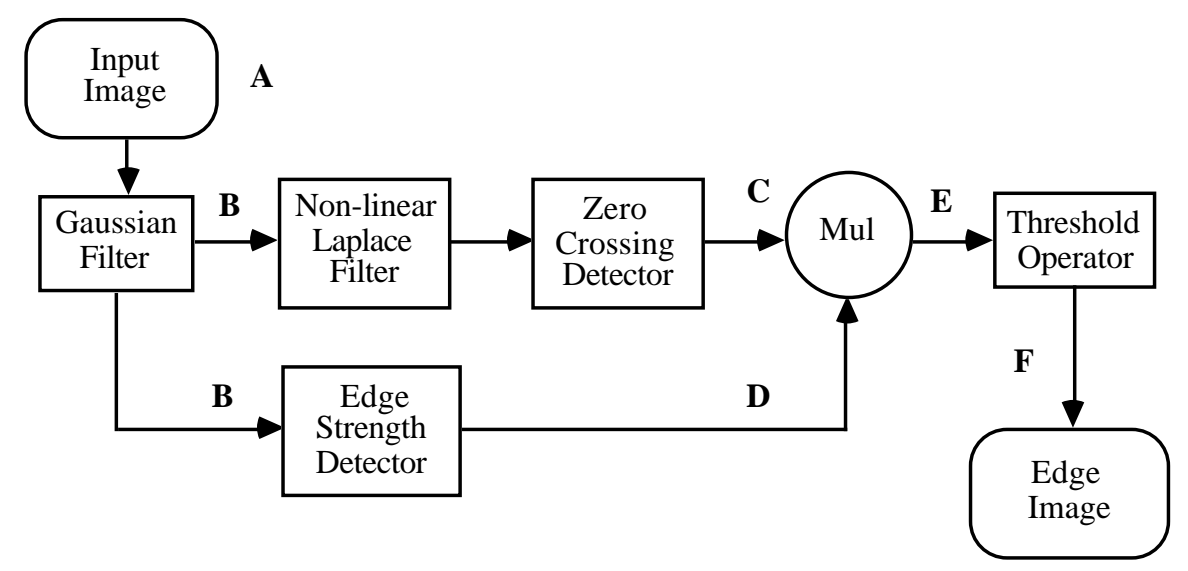

Figure 1: The edge detection model. 


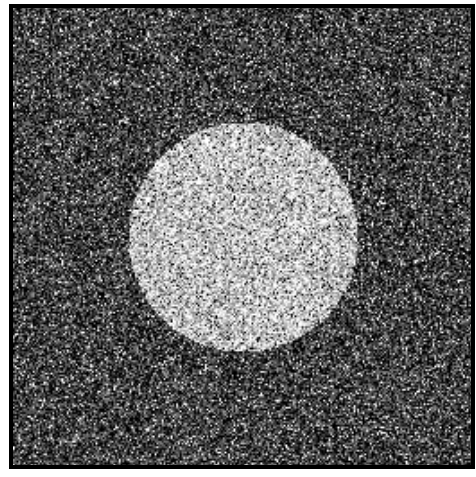

a

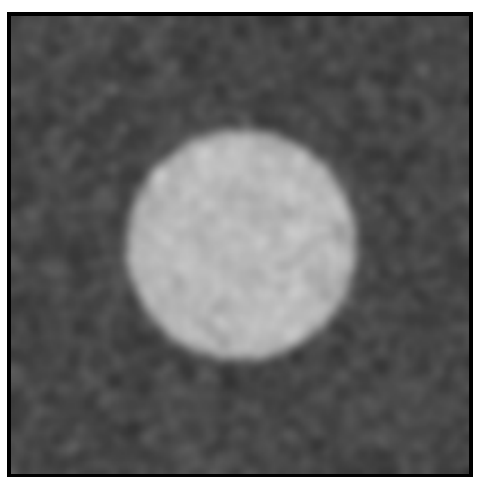

b

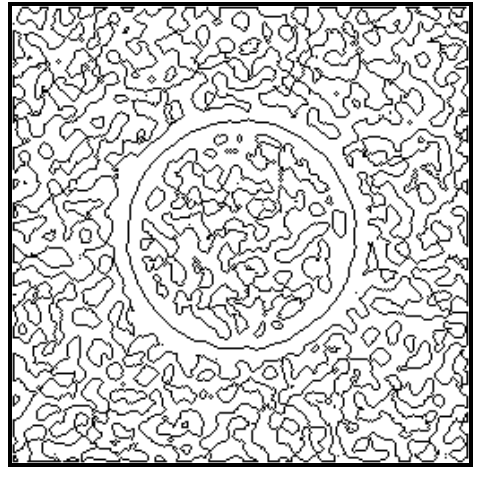

c

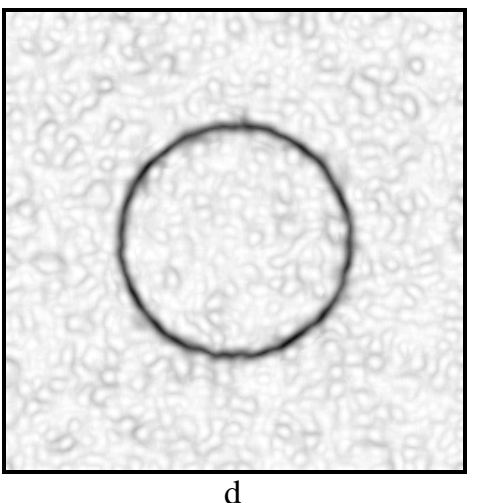

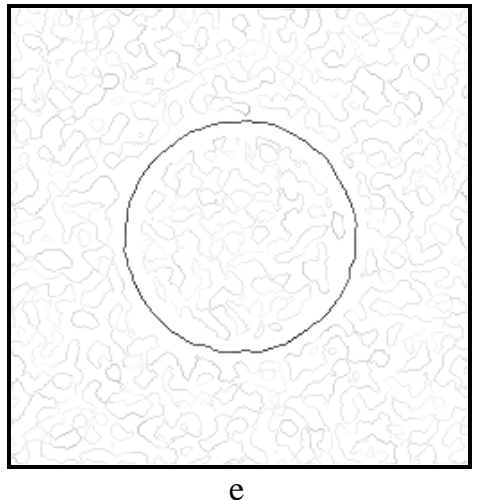

e

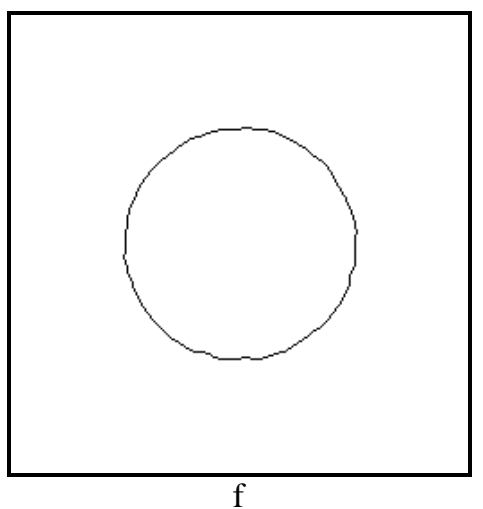

Figure 2: Pictures showing the intermediate results during the various stages of the edge detection process. The character code corresponds to the marked places in the flow diagram of the edge detection model of fig. 1 .

\subsection{Smoothing Filter}

A Smoothing filter is applied to a 'noisy' input image in order to suppress the noise level using a form of averaging. A uniform filter of size $n\left(n^{2}\right.$ samples) reduces the noise variance by a factor $\mathrm{n}^{2}$, thus increasing the signal-to-noise ratio. Another smoothing filter is a Gaussian filter. Even if the noise suppressing factor of these two filters are the same, the frequency responses remain completely different. Gaussian smoothing is superior to uniform smoothing which can be shown both analytically (Marr [1]; Canny [5]) and experimentally (Van Vliet [6]). The smoothing filter should, however, be as small as possible because averaging blurs the image and decreases resolution.

\subsection{Non-linear Laplace Operator}

Laplace filters used in image processing are digital approximations of their mathematical equivalent; a variety of such filters can be constructed. A $3 \times 3$ filter which is frequently used is given in eq.(1).

$$
\nabla^{2} \mathrm{I}(\mathrm{x}, \mathrm{y}) \approx \mathrm{I}(\mathrm{x}+1, \mathrm{y})+\mathrm{I}(\mathrm{x}-1, \mathrm{y})+\mathrm{I}(\mathrm{x}, \mathrm{y}+1)+\mathrm{I}(\mathrm{x}, \mathrm{y}-1)-4 \mathrm{I}(\mathrm{x}, \mathrm{y})
$$

The Laplacian is rotation invariant which means that spatial frequencies in all directions are equally enhanced. Unfortunately, it cannot distinguish signal and noise which makes it a useless tool in images with medium and low signal-to-noise ratios (Ballard [2]). Directional information is not available as well. 
A possible solution to these disadvantages of the linear Laplace filter is the design of a non-linear Laplace filter, a non-linear combination of neighborhood pixels. Our non-linear Laplace filter has two filter parameters: size and shape. For a more detailed description of the non-linear Laplace operator of eq.(2) we refer to Van Vliet et. al.[6].

$$
\operatorname{NLLAP}(\mathrm{x}, \mathrm{y})=\operatorname{gradmax}(\mathrm{x}, \mathrm{y})+\operatorname{gradmin}(\mathrm{x}, \mathrm{y})
$$

where

$$
\begin{aligned}
& \operatorname{gradmax}(\mathrm{x}, \mathrm{y})=\max \left\{\operatorname{Cn}\left(\mathrm{x}^{\prime}, \mathrm{y}^{\prime}\right)\left[\mathrm{I}\left(\mathrm{x}^{\prime}, \mathrm{y}^{\prime}\right)-\mathrm{I}(\mathrm{x}, \mathrm{y})\right] \mid\left(\mathrm{x}^{\prime}, \mathrm{y}^{\prime}\right)-\mathrm{d}_{\mathrm{n}}(\mathrm{x}, \mathrm{y})\right\} \\
& \operatorname{gradmin}(\mathrm{x}, \mathrm{y})=\min \left\{\mathrm{Cn}\left(\mathrm{x}^{\prime}, \mathrm{y}^{\prime}\right)\left[\mathrm{I}\left(\mathrm{x}^{\prime}, \mathrm{y}^{\prime}\right)-\mathrm{I}(\mathrm{x}, \mathrm{y})\right] \mid\left(\mathrm{x}^{\prime}, \mathrm{y}^{\prime}\right) \mathrm{d}_{\mathrm{n}}(\mathrm{x}, \mathrm{y})\right\}
\end{aligned}
$$

where $\mathrm{d}_{\mathrm{n}}(\mathrm{x}, \mathrm{y})$ is an $\mathrm{nxn}$ square centered at $(\mathrm{x}, \mathrm{y})$ and $\mathrm{C}_{\mathrm{n}}\left(\mathrm{x}^{\prime}, \mathrm{y}^{\prime}\right)$ is a multiplication mask consisting of scale coefficients dependent on the filter size and the desired filter shape, i.e. circular or square. For $\mathrm{C}_{\mathrm{n}}\left(\mathrm{x}^{\prime}, \mathrm{y}^{\prime}\right)=\left\{1 \mid\left(\mathrm{x}^{\prime}, \mathrm{y}^{\prime}\right) \in \mathrm{d}_{\mathrm{n}}(\mathrm{x}, \mathrm{y})\right\}$ eq. (2) can be simplified to:

$$
\begin{aligned}
& \operatorname{gradmax}(\mathrm{x}, \mathrm{y})=\operatorname{MAX}_{\mathrm{n}}\{\mathrm{I}(\mathrm{x}, \mathrm{y})\}-\mathrm{I}(\mathrm{x}, \mathrm{y}) \\
& \operatorname{gradmin}(\mathrm{x}, \mathrm{y})=\operatorname{MIN}_{\mathrm{n}}\{\mathrm{I}(\mathrm{x}, \mathrm{y})\}-\mathrm{I}(\mathrm{x}, \mathrm{y})
\end{aligned}
$$

where $\mathrm{MAX}_{\mathrm{n}}$ and $\mathrm{MIN}_{\mathrm{n}}$ are two simple non-linear filters, the local maximum and the local minimum filter with an nxn square window as structuring element.

Non-square filters can be built in one of two ways. Circular filter shapes can be approximated by chosing the proper multiplication masks $C_{n}\left(x^{\prime}, y^{\prime}\right)$. These masks can be constructed by rescaling the mask coefficients lying outside an imaginary circle with radius (n-1)/2 (n represents the size of the non-linear Laplace operator). Examples of those masks for $n=3,5$, and 7 are given by Van Vliet [6]. In addition, mask coefficients which originate completely from outside this circle may be set to zero. Alternatively, arbitrary filter shapes can be constructed by taking maximum and minimum filters of such shapes. The impact of several shapes: square, diamond, circular, circular/hollow on edge detection is presented by Verbeek et al. [7]. Fast algorithms for these filters on general purpose computers are given as well.

Concluding, the non-linear Laplace operator has the following potential advantages:

- adaptive orientation perpendicular to the local edge direction;

- decreased sensitivity to noise;

- simple implementation.

\subsection{Zero Crossing Detector}

A zero-crossing detector assigns a label 'edge' to these locations where the Laplace filtered image changes sign. It will be very unlikely that zero-crossings coincide with our spatial coordinates called pixels. The detection of those zero-crossings can be done very accurately by subpixel interpolation techniques. Subpixel precision can also be achieved by parametric methods. A least square estimator is used to compute locally the parameters of a set of discrete polynomials which describe the underlying grey values. A precision less than 0.5 pixel can be achieved by a nearest neighbor approach. A disadvantage of the above mentioned techniques is a required boundary condition to guarantee the connectedness of the edge pixels. Such conditions slow down the speed of those algorithms.

Our fast zero-crossing detector solves these problems in exchange for a maximum displacement of 1 pixel (fig. 3). After an initial segmentation into three regions: positive, zero, and negative, the zero value pixels are assigned to the nearest adjacent region using two distance transformations 
(Borgefors [8]). This guarantees a good performance when dealing with blurred images where long ramp edges often occur. From the resulting binary image (positive, negative) the 8-connected contour is extracted. This is a very important step because closed contours are found without additional computational effort.

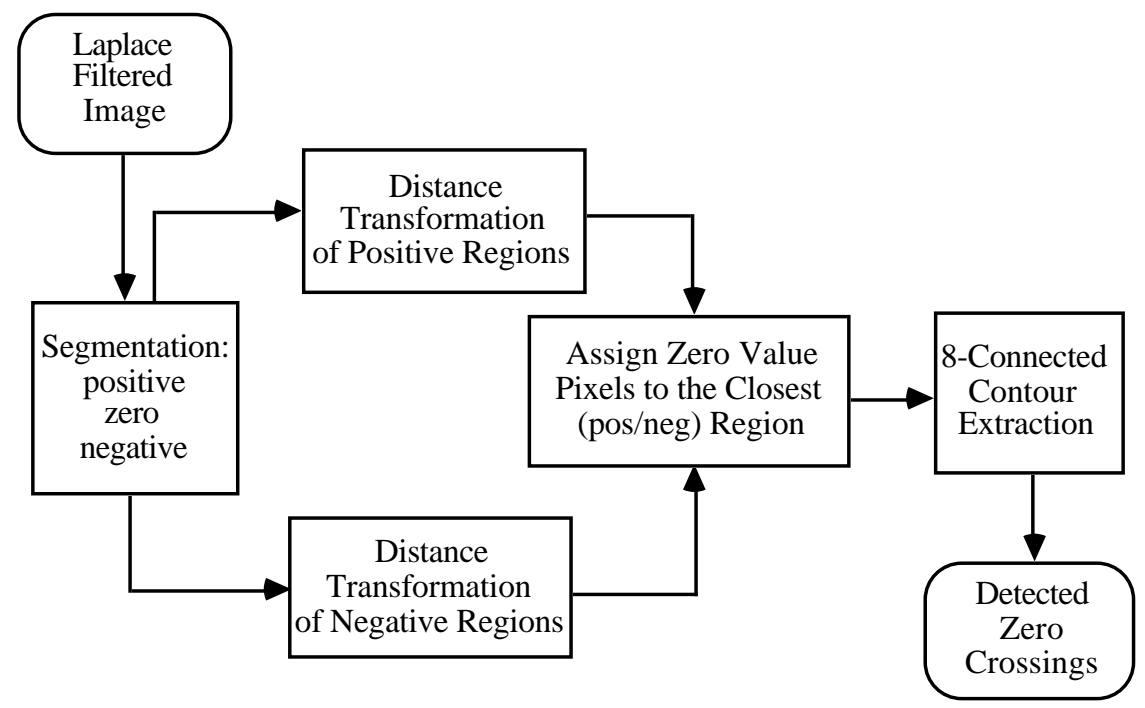

Figure 3: The zero-crossing detection scheme.

\subsection{Edge Strength Detector}

The edge strength at an arbitrary location is a measure for the steepness of an edge at that location in the input image. Gradient-like filters are able to provide edge strength images. Comparative studies by Abdou [9] and Peli [10] have pointed out, however, that common gradient operators such as Roberts [11], Prewitt [12], and Sobel [13] perform poorly in images with medium and low signalto-noise ratios.

Lee's [14] morphologic edge detector is less noise sensitive and performs optimal on blurred images (fig. 4). A step edge can only be detected after the image has been smoothed. Figure 4 shows that the ridges in Lee's edge strength image coincide with the zero-crossings of our non-linear Laplace operator. In this detector we notice a strong parallel with the non-linear Laplace operator. Lee's definition can be rewritten in terms of gradmax and gradmin as follows:

$$
\operatorname{IEdge}-\operatorname{Strength}(\mathrm{x}, \mathrm{y})=\min \{\operatorname{gradmax}(\mathrm{x}, \mathrm{y}),-\operatorname{gradmin}(\mathrm{x}, \mathrm{y})\}
$$

The above given equation implies a reduction in computational effort. Namely, gradmax and gradmin have to be computed only once and are used twice.

\section{EVALUATION PROCEDURE}

The model described in the preceding section has parameters associated with it: the standard deviation of the Gaussian filter; and the size and effective shape of both the non-linear Laplace operator and the edge strength filter. In order to take advantage of the parallel between the last two, the size and shape of both filters are chosen equal. 


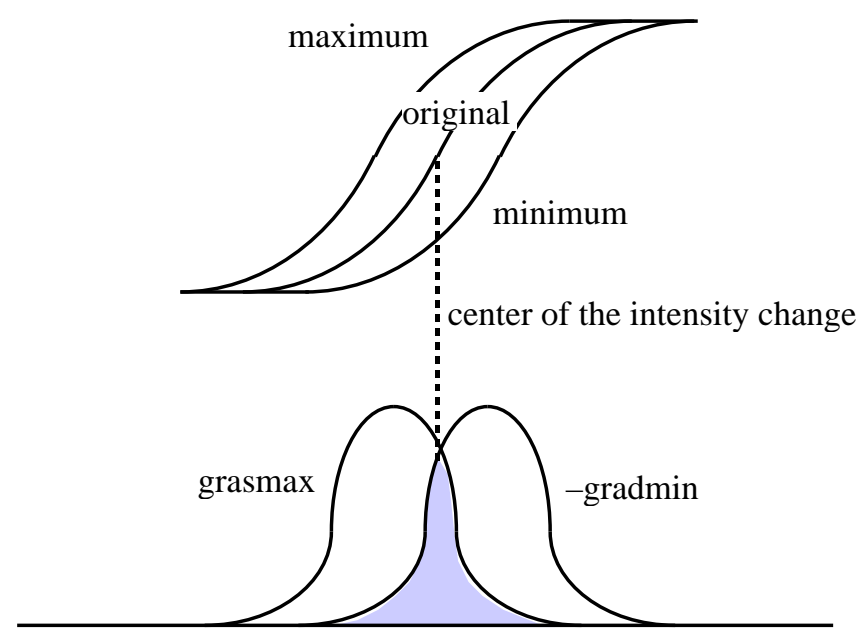

Figure 4: Lee's blur minimum morphologic edge detector is very effective on a blurred edge profile.

In our research we studied the relation between the SNR of the input image and the parameter choices of the edge detection model, in particular the Gaussian filter size and the non-linear Laplace filter size versus the SNR. The SNR is defined as:

$$
S N R=\frac{g^{2}}{\sigma_{n}^{2}}
$$

where $g$ is the grey level difference between the two sides of an edge, and $\sigma_{\mathrm{n}}$ the standard deviation of the additive independent Gaussian noise with zero mean. Within a small range of SNR, the optimum configuration of our edge detector ought to be the one with the smallest support in the spatial domain. As an objective quantitative performance measure we have chosen Pratt's figure of merit (Pratt [4]).

$$
F O M=\frac{1}{\max \left(I_{D}, I_{I}\right)} \sum_{i=1}^{I_{D}} \frac{1}{1+\alpha d_{i}^{2}}
$$

where $I_{D}$ is the number of detected edge points, II the number of ideal edge points (ground truth), $\alpha\left(>0\right.$, often 1/9) a scaling factor, and $d_{i}$ the edge deviation or error distance for the $\mathrm{i}$ th detected edge pixel.

Pratt's figure-of-merit has often been used in edge detection evaluation which allows us to compare our results with those of others in case the test images are the same. However, Pratt's figure-ofmerit strongly depends on the threshold level used to obtain a binary edge image. In the literature (Peli [10]) the threshold level was set to achieve the maximum figure-of-merit. We chose instead for thresholds that provided closed contours and took our chances with the resulting figure-of-merit. We did so, because the edge results with maximum figure-of-merit consist of many small disconnected pieces from which hardly any information can be derived. Unfortunately, Pratt's figure-of-merit gives no bonus for finding closed contours and small eroneous fragments far from the true contour are heavily penalized. 


\section{EXPERIMENTAL RESULTS}

In order to estimate the optimal parameter choice for a wide range of SNRs $(1 \leq \mathrm{SNR} \leq 100)$ we divide the SNR interval in two parts: $1 \leq \mathrm{SNR} \leq 10$ and $10<\mathrm{SNR} \leq 100$. This is done for display purposes. In our model we used a Gaussian smoothing filter, a non-linear Laplace filter, and Lee's edge strength filter, all with a pseudo circular shape and of different sizes. We used a circular test image with additive independent Gaussian noise with zero mean (fig. 2a). Figure 5 shows the figure-of-merit for several values of $\sigma$ (standard deviation of a Gaussian filter) and $n$ (filter size of a non-linear Laplace filter) over the entire range of SNRs.
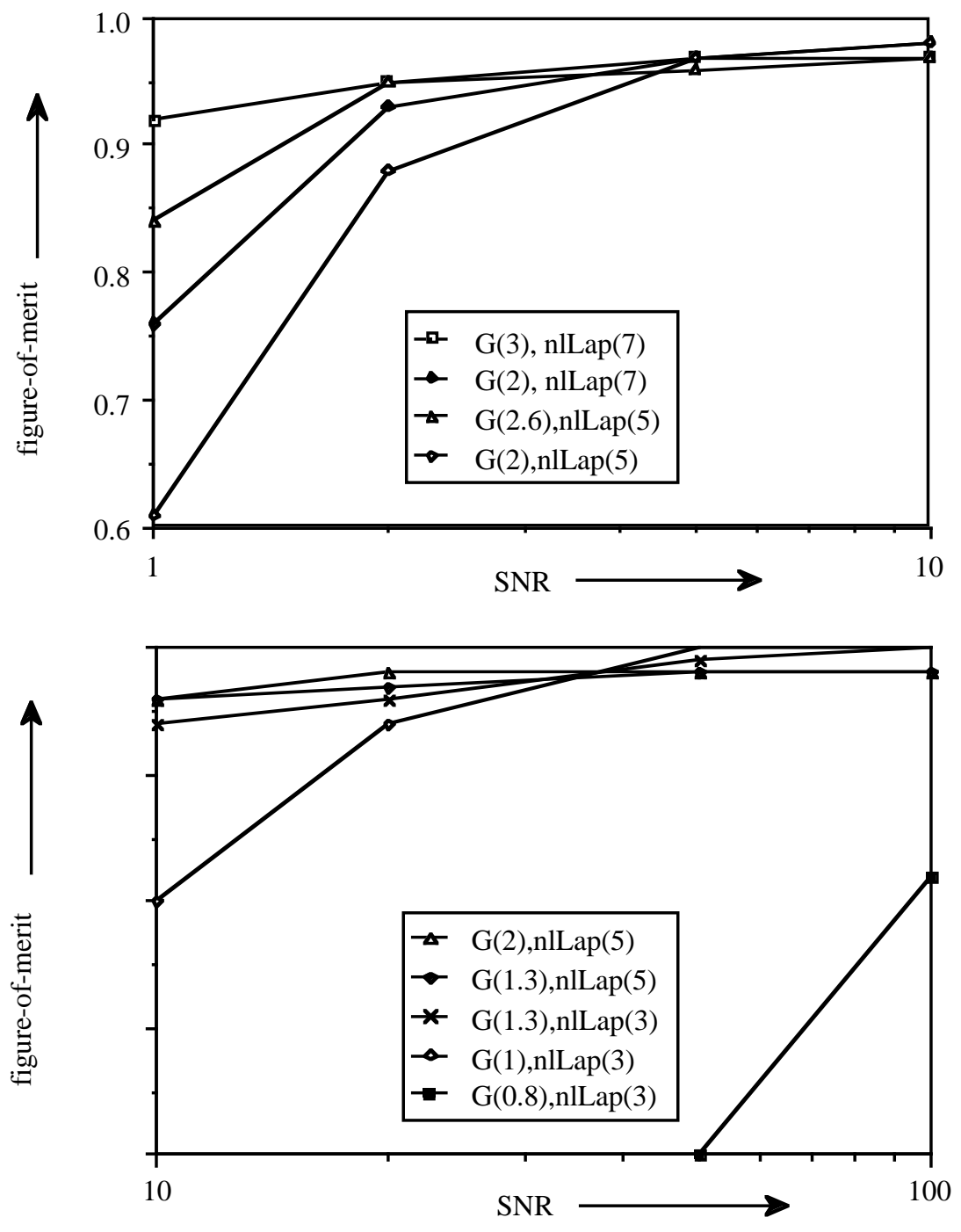

Figure 5: The obtained figure-of-merits of several configuration of the edge detection model as function of the signal-to-noise ratio. A synthetic image containing a circle with additive independent Gaussian noise with zero mean is used. All non-linear Laplace filters as well as edge strength operators have a circular support. 
From figure 5 we may deduce that there is a lower bound for the amount of smoothing somewhere between $\sigma=0.8$ and $\sigma=1.0$. As the SNR decreases we observe a monotonic increase in the standard deviation of the Gaussian filter required to achieve a respectable figure-of-merit. The optimum size of the non-linear Laplace filter increases as well. Figure 6 shows a rough estimate of the locally optimal values for $\sigma$ and $\mathrm{n}$ as a function of the SNR. The grey region indicates the uncertainty associated with these parameter values.

Examining the above results we should not forget that the test object is relatively large in relation to the total filter size. If the complexity of the image is greater with more spatial details, the size of the filters used can interfere with the estimation of the true edge positions. This is illustrated in the rounded corners of the detected edges in the highly structured shawl of figure $7 \mathrm{~b}$. Very fine spatial details embedded in a high noise level cannot be detected or are only partially be detected.

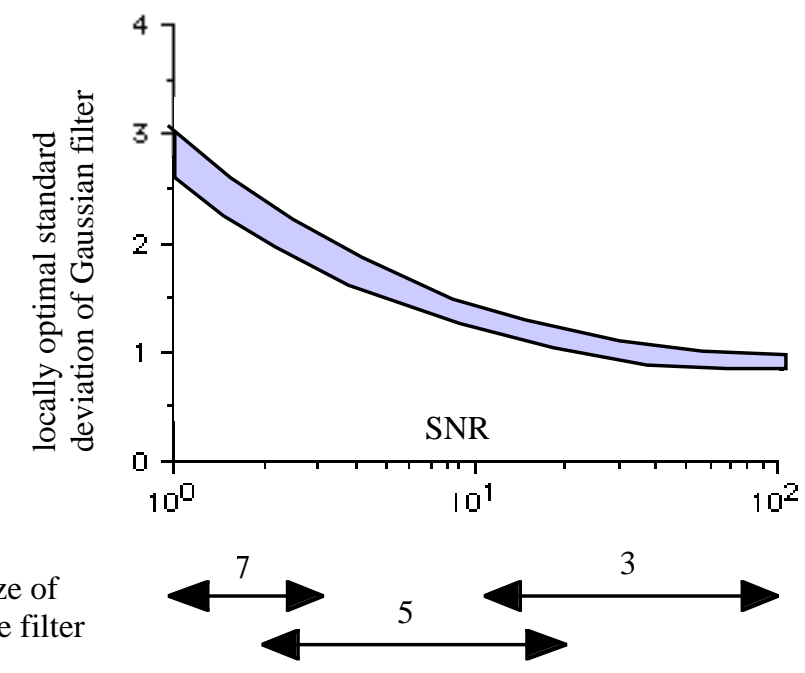

Figure 6: Locally optimal standard deviation of the Gaussian smoothing filter and locally optimal size of the non-linear Laplace filter as function of the signal-to-noise ratio.

\section{COMPARISONS}

In the previous section we have examined the influence of the parameters $\sigma$ and $n$ on our edge detection scheme. This has resulted in a locally optimal configuration as function of the SNR in the input image. This was done for a circular test image with additive independent Gaussian noise. Peli(1982) reported an extensive evaluation of well known edge detectors on a similar test image. His results are shown in Peli [10] figure 11b. He concludes that the Roberts' gradient is a useful edge detector in images with high SNR due to its computational simplicity while the Rosenfeld [15-17] algorithm performs better in noisy images. The idea behind the latter technique is that of computing differences between averages of non-overlapping neighborhoods that meet at the same point. However, our figure 5 compared with Peli (fig. 11b) shows that the model proposed here is superior to these other techniques for the entire range of SNR. 


\section{CONCLUSIONS}

An edge detection scheme inspired by the Marr-Hildreth edge detection model has been described and evaluated. The scheme reserves a central role for the non-linear Laplace operator and is, therefore, extremely effective and flexible in the detection of one pixel thick edges. We have empirically determined the relation between the SNR of the input image and the parameters of the edge detection model, resulting in a locally optimal configuration for a wide range of SNRs $(1 \leq$ SNR $\leq 100)$.

Comparisons with other edge detectors based on Pratt's figure-of-merit shows that our model perform at least as well, and in most cases far better, than all other techniques (Peli [10], Van Vliet [6]).

Finally we present in figure 7 the edge results achieved by applying our detector to some "real world" images.
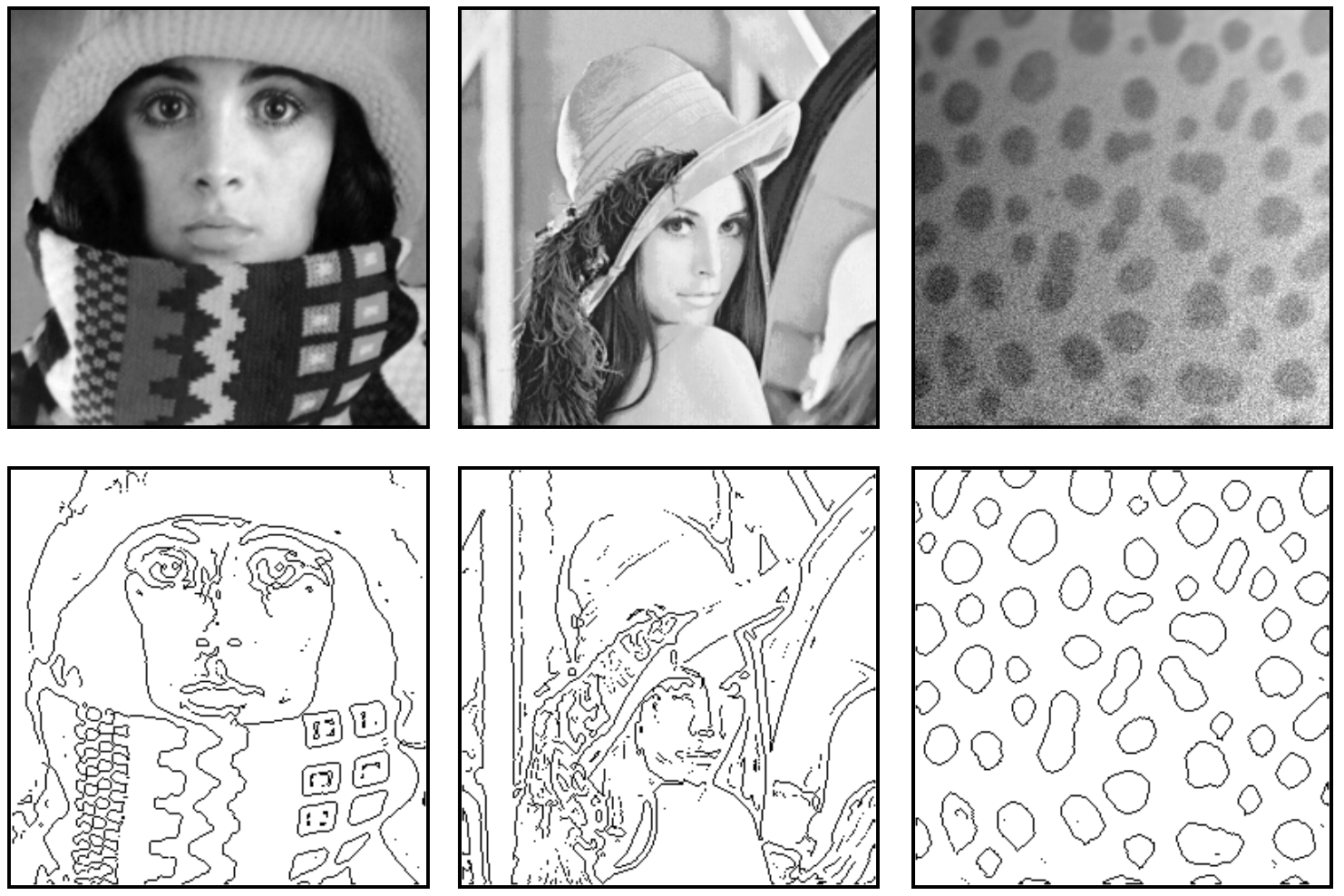

Figure 7: Presentation of the edge results achieved by applying our edge detector to "real world" images. In the shawl of bottom-left figure we notice a displacement of the detected edge position at sharp corners, caused by interference between these small spatial details and the size of the edge detector.

\section{ACKNOWLEDGEMENT}

The authors thank Dr. Piet Verbeek and Ir. Henri Vrooman for their valuable comments during various stages of this project. The research was done at the Pattern Recognition Group of the Faculty of Applied Physics at the Delft University of Technology, The Netherlands under partial 
sponsorship of The Netherlands foundation for Medical Research, MEDIGON, grant 900-528-016 and the Netherlands Foundation for Fundamental Research on Materials, FOM, grant DTN95.0329.

\section{REFERENCES}

[1] Marr, D. and E.C. Hildreth, Theory of edge detection, Proc. R. Soc. London Ser. B. 207, 1980, pp. 187-217.

[2] Ballard, D.A. and C.M. Brown, Computer Vision, Prentice-Hall Inc., Englewood Cliffs, 1982.

[3] Beckers, A.L.D., Metingen van Parameters voor Niet-lineaire Objectgrootte-filters in beelden, Master's thesis, Department of Applied Physics, Delft University of Technology, 1986.

[4] Pratt, W.K., Digital Image Processing, Wiley, New York, 1977.

[5] Canny, J., A Computational Approach to Edge Detection, IEEE Trans. Pattern Anal. Machine Intell., PAMI-8, 1986, pp. 679-698.

[6] Van Vliet, L.J., I.T. Young, and A.L.D. Beckers, A Nonlinear laplace Operator as Edge Detector in Noisy Images, Submitted to Computer Vision, Graphics and Image Processing, 1988.

[7] Verbeek, P.W., H.A. Vrooman, and L.J. van Vliet, Low Level Image Processing by Max-Min Filtering, accepted by Signal Processing, 1988.

[8] Borgefors, G., Distance Transformations in Arbitrary Dimensions, Computer Vision, Graphics and Image Processing, Vol.27, 1984, pp. 321-345.

[9] Abdou, I.E. and W.K. Pratt, Quantitative Design and Evaluation of Enhancement/ Thresholding Edge Detectors, Proc. IEEE, Vol. 67, No.5, 1979, pp. 753-763.

[10] Peli, T. and D. Malah, A Study on Edge Detection Algorithms, Computer Graphics and Image Processing, Vol.20, 1982, pp. 1-21.

[11] Roberts, L.G., Machine Perception of three Dimensional solids, Optical and electro-optical Information Processing, J.T. Tippet et al., (Eds.), Cambridge, MA: M.I.T. Press, 1965, pp. 159-197.

[12] Prewitt, J.M.S., Object Enhancement and Extraction, Picture processing and Psychopictorics, B.S. Lipkin and A. Rosenfeld, Eds. New York: Academic Press, 1970.

[13] Duda, R.O. and P.E. Hart, Pattern Classification and Scene Analysis, Wiley, New york, 1973, pp. 271-272.

[14] Lee, J.S.L., R.M. Haralick and L.S. Shapiro, Morphologic Edge Detection, Proc. 8th Inter. Conf. Pattern Recognition, Paris (France), 1986, pp. 369-373.

[15] Rosenfeld, A., A Nonlinear Edge Detection Technique, Proc. IEEE, Vol.58, 1970, pp. 814816.

[16] Rosenfeld, A., and M Thurston, Edge and Curve Detection for Visual Scene Analysis, IEEE Trans. Comput., C-20, 1971, pp. 562-569.

[17] Rosenfeld, A., M Thurston and Y.H. Lee, Edge and Curve Detection: Further Experiments, IEEE Trans. Comput., C-21, 1972, pp. 677- 15. 